\title{
Comparação entre métodos magistrais de homogeneização para a preparação de cápsulas e alendronato de sódio: saco plástico $x$ almofariz
}

\author{
Comparison between homogenization methods for compounded \\ sodium alendronate capsules: plastic bag x mortar and pestle
}

\author{
Mayara Myuki WADA, Ana Ferreira RIBEIRO \\ Instituto Federal de Educação, Ciência e Tecnologia do Rio de Janeiro, \\ Campus Realengo. Rua Professor Carlos Wenceslau, 343, Realengo. \\ CEP 21715-000 - Rio de Janeiro, RJ-Brasil
}

\begin{abstract}
Homogenization using mortar and pestle is a traditional method for powder mixing of drugs formulations. Compounding pharmacies have used a method of homogenization using plastic bags inflated with air in order to improve the production of capsules. In this work the efficiency of this method compared to the mortar and pestle one, for the production of sodium alendronate capsules, was evaluated. Batches of capsules containing $70 \mathrm{mg}$ of alendronic acid (equivalent to $91.42 \mathrm{mg}$ of sodium alendronate trihydrate) were produced using, for the homogenization of powder blend, the mortar and pestle technique (ALE-MP) and the plastic bag method with or without the previous sieving of the bulk materials (ALE-PB-S and ALE -PB, respectively). Acid-base titration method was used for the quantitative determination of alendronate in the capsules. Each batch was evaluated with respect to drug content, uniformity of mass and content uniformity and presented results according to the pharmacopeial specifications. However, after analysis of the content uniformity data, we found a statistically significant difference in the variance of the ALE-PB batch compared to the other batches, mainly the ALE-MP batch $(\mathrm{P}<0.0001$, for $\alpha=0.05)$. This result suggests poor homogenization efficiency for the plastic bag method without sieving.
\end{abstract}

Key Words: sodium alendronate, powder mixture, content uniformity

\section{RESUMO}

A homogeneização em almofariz é o método tradicional de mistura de pós, usado na manipulação magistral de cápsulas. Para melhorar o processo de produção, farmácias magistrais tem utilizado um método de mistura alternativo utilizando sacos plásticos inflados com ar. O objetivo deste trabalho foi avaliar a eficiência desta técnica em comparação ao método do almofariz, para a produção de cápsulas de alendronato de sódio. Foram produzidas cápsulas contendo $70 \mathrm{mg}$ de ácido alendrônico por unidade (equivalentes a 91,42 mg de alendronato de sódio triidratado), empregando, para a mistura dos pós, o método de homogeneização em almofariz (ALE-A) e o método do saco plástico com tamisação prévia (ALE-SP-T) e sem tamisação prévia dos componentes (ALE-SP). Para a análise quantitativa da substância ativa nas cápsulas foi utilizado método titulométrico ácido-base. Cada lote foi avaliado com relação ao teor médio, peso médio e uniformidade de conteúdo, apresentando resultados de acordo com as especificações farmacopeicas. No entanto, após análise estatística dos dados de uniformidade de conteúdo, encontramos uma diferença significativa entre a variância do lote ALE-SP e a dos outros lotes, principalmente em comparação ao lote ALE-A ( $\mathrm{P}<0,0001$, para $\alpha=0,05)$. Este resultado sugere baixa eficiência de homogeneização para o método do saco plástico sem tamisação prévia das matérias-primas.

Palavras-Chave: aendronato de sódio, mistura de pós, uniformidade de conteúdo 


\section{INTRODUÇÃO}

A homogeneização em gral com pistilo é o método de mistura de pós para cápsulas tradicionalmente utilizado em estabelecimentos magistrais e que se encontra descrito em diversas literaturas (1-3)

No entanto, há alguns anos, as farmácias magistrais desenvolveram técnicas alternativas de homogeneização, visando obter maior rapidez e praticidade durante o processo de manipulação. A utilização de recipientes plásticos para a mistura de pós apresenta-se como uma técnica bastante atraente neste sentido e que vem sendo adotada muito frequentemente em um grande número de farmácias magistrais (4).

Independente da técnica de manipulação utilizada é importante conferir especial atenção à qualidade de homogeneização proporcionada, a qual deve resultar em uma mistura uniforme entre fármaco e excipientes e, consequentemente, permitir uma adequada uniformidade das doses a serem administradas em cada cápsula $(2,4)$.

Diversos estudos mencionam os principais fatores que afetam de maneira significativa a uniformidade de uma mistura de pós (5-12). Entre estes, podemos destacar a distribuição do tamanho das partículas, o tempo e a velocidade de mistura, os adjuvantes empregados e a proporção entre fármaco e excipientes.

Além disto, o processo de obtenção da forma farmacêutica final, seja ela uma cápsula ou um comprimido, pode também influenciar na qualidade do produto em relação à sua uniformidade de doses $(13,14)$.

Usualmente, para avaliar a eficiência de homogeneização, são empregados testes farmacopeicos para a determinação do teor em pontos distintos da mistura de pós, ou mesmo para a determinação da uniformidade de doses das unidades farmacêuticas. Em farmácias magistrais, realizar este tipo de análise em todas as formulações manipuladas torna-se inviável. Assim, tais determinações devem ser realizadas a cada 2 ou 3 meses, em formulações contendo baixa concentração de fármaco em relação aos excipientes, como é o caso dos diluídos e de medicamentos com dose do fármaco inferior a $25 \mathrm{mg}$ (15). Considerando a baixa frequência de realização destas análises, garantir a uniformidade de doses das cápsulas depende, principalmente, de um procedimento eficaz e padronizado para a manipulação deste tipo de medicamento (16).

Sendo assim, torna-se necessário avaliar a eficiência de homogeneização das técnicas alternativas de mistura que vêm sendo empregadas nas farmácias magistrais, como é o caso da homogeneização em recipiente plástico. Com isto, pode-se evidenciar a necessidade de uma padronização dos procedimentos adotados, visando validar a técnica e harmonizá-la entre os vários estabelecimentos de manipulação.

Um tipo de prescrição na forma sólida, aviada com grande frequência nos estabelecimentos magistrais, é a formulação de cápsulas de alendronato de sódio. Este fármaco é utilizado principalmente no tratamento de desordens relacionadas ao tecido ósseo, devido à sua capacidade de inibir o processo de reabsorção óssea mediada pelos osteoclastos (17-18). As dosagens mais comuns para cápsulas de alendronato de sódio são de $10 \mathrm{mg}$ e 70 mg de ácido alendrônico por cápsula, sendo esta última a mais utilizada pelos prescritores.

O alendronato de sódio é um composto aminodifosfonado que possui em sua estrutura um grupamento ácido, o qual possibilita sua titulação por hidróxido de sódio (19-20). Embora existam diversas metodologias descritas para análise do alendronato de sódio, empregando desde a espectrofotometria e a cromatografia líquida de alta eficiência, até métodos menos comuns na rotina laboratorial (21), a titulometria é, sem dúvida, o método preferencialmente utilizado em estabelecimentos magistrais, devido à sua simplicidade e baixo custo.

Neste trabalho foi verificada a eficiência de homogeneização da mistura de pós para cápsulas manipuladas de alendronato de sódio, contendo $70 \mathrm{mg}$ de ácido alendrônico por cápsula (equivalentes a 91,42 mg de alendronato de sódio triidratado), através da avaliação do método alternativo de homogeneização em saco plástico em comparação ao método de homogeneização em almofariz. A titulometria de neutralização foi utilizada para a quantificação do alendronato nas cápsulas, já que o método foi validado para a formulação analisada (22).

\section{MATERIAL E MÉTODOS}

\section{Obtenção das cápsulas de alendronato de sódio}

Para o preparo das cápsulas, foram utilizadas as matérias-primas de grau farmacêutico alendronato de sódio e dióxido de silício coloidal (Aerosil ${ }^{\circledR}$ ), fornecidas pela Audaz Reagentes Tecnológicos (São Paulo, Brasil), lactose adquirida da Casa da Química Indústria e Comércio LTDA (Diadema, Brasil) e cápsulas de gelatina dura número 1, da Capsugel $^{\circledR}$ (Capsugel, Bornem, Bélgica).

Para as análises efetuadas foram utilizados os reagentes de grau analítico hidróxido de sódio e fenolftaleína, obtidos da Audaz Reagentes Tecnológicos e biftalato de potássio, adquirido da Cromato Produtos Químicos (Diadema, Brasil). 


\section{Avaliação do alendronato de sódio matéria-prima}

O alendronato de sódio, utilizado como matéria-prima para o preparo dos lotes de cápsulas, foi analisado quanto à identificação e teor de pureza. Para a identificação foram realizados testes para sódio e fosfato (23). Para a avaliação do teor foi utilizada titulometria de neutralização, com emprego de hidróxido de sódio $0,1 \mathrm{~mol} / \mathrm{L}$ (SV) como titulante e determinação visual do ponto de equivalência utilizando fenolftaleína (20).

\section{Determinação do peso médio das cápsulas}

Para a determinação do peso médio, foram separadas 20 cápsulas de cada lote. Cada cápsula foi pesada em balança analítica (Mettler Toledo ${ }^{\circledR}$ AG204) e depois esvaziada, pesando-se o invólucro vazio em seguida. Desta forma, foi calculado o conteúdo de pó em cada cápsula, repetindo-se este procedimento para cada uma das 20 unidades selecionadas (23).

\section{Determinação do teor médio das cápsulas}

Para a determinação do teor médio de cada lote, o conteúdo das 20 cápsulas analisadas no peso médio foi homogeneizado em gral com pistilo. Uma alíquota deste pó foi pesada e o teor avaliado por titulação com hidróxido de sódio $0,1 \mathrm{~mol} / \mathrm{L}$ (SV) (22).

\section{Determinação da uniformidade de conteúdo das cápsulas}

Para a determinação da uniformidade de conteúdo, no mínimo 20 cápsulas de cada lote foram avaliadas, individualmente, de acordo com métodos oficiais recomendados para a realização deste teste $(23,24)$. $O$ teor de alendronato de sódio foi determinado titulando-se cada cápsula com hidróxido de sódio $0,05 \mathrm{~mol} / \mathrm{L}$ (SV) e determinando-se visualmente o ponto de equivalência (20). Para realizar a comparação entre as variâncias dos diferentes lotes, os resultados de uniformidade de conteúdo foram submetidos ao teste de Fisher (distribuição F), utilizando o programa GraphPad Prism ${ }^{\circledR}$ (versão 5.0, GraphPad Software Inc., EUA).

\section{RESULTADOS E DISCUSSÃO}

\section{Manipulação das cápsulas de alendronato de sódio \\ Preparação da formulação ALE-A, utilizando método de mistura em almofariz}

Cada matéria-prima foi pesada exatamente e em quantidade suficiente para o preparo de 1 lote (Tabela 1) e a homogeneização foi realizada por 2 minutos em almofariz, seguida da encapsulação em cápsulas de gelatina dura número 1 (volume $=0,48 \mathrm{~mL}$ ). Este procedimento foi executado em dois dias consecutivos, resultando nos lotes ALE-A1 e ALE-A2.
Tabela 1 - Composição de cada lote contendo 60 cápsulas de alendronato de sódio

\begin{tabular}{|l|c|}
\hline \multicolumn{1}{|c|}{ Componente } & Quantidade \\
\hline Alendronato de sódio & $5,4852 \mathrm{~g}$ \\
\hline Lactose & $14,1486 \mathrm{~g}$ \\
\hline Aerosil & $0,0982 \mathrm{~g}$ \\
\hline
\end{tabular}

Preparação da formulação ALE-SP, utilizando método de mistura em saco plástico

Separadamente foi pesada, exatamente, quantidade suficiente de matéria-prima para o preparo de 1 lote, conforme a Tabela 1. Os componentes foram homogeneizados por 2 minutos em saco plástico, através da agitação em vários sentidos, e a mistura de pós foi encapsulada em cápsulas número 1 . Este procedimento foi executado em dois dias consecutivos, resultando nos lotes ALE-SP1 e ALE-SP2.

\section{Preparação da formulação ALE-SP-T,} utilizando método de mistura em saco plástico, com tamisação prévia das matérias-primas

Cada matéria-prima foi previamente peneirada através de tamis de aço inoxidável malha 20 e em seguida pesada exatamente, em quantidade suficiente para o preparo de 1 lote, (Tabela 1). Foi realizada homogeneização em saco plástico por 2 minutos, através da agitação em vários sentidos, e a mistura de pós foi encapsulada em cápsulas de gelatina número 1. Este procedimento foi realizado em dois dias consecutivos, resultando nos lotes ALE-SP-T1 e ALE-SP-T2.

\section{Avaliação do alendronato de sódio matéria prima}

Nos ensaios de identificação realizados, a matéria-prima apresentou reação positiva para sódio e para grupamento fosfórico, de acordo com o esperado para a molécula de alendronato de sódio. $\mathrm{Na}$ avaliação da pureza obteve-se o teor médio de $100,29 \%$ e desvio padrão relativo (DPR) de $0,82 \%$ entre as análises $(n=4)$.

\section{Determinação do teor médio das cápsulas}

Os valores de teor médio encontrados para todas as formulações podem ser visualizados na Tabela 2.

Tabela 2 - Valores de teor médio encontrados paracada método de homogeneização de pós para a manipulação de cápsulas de alendronato de sódio $(\mathrm{n}=8)$.

\begin{tabular}{|c|c|c|}
\hline Formulação & Teor Médio (\%) & DPR (\%) \\
\hline ALE-A & 98,6 & 1,15 \\
\hline ALE-SP-T & 102,7 & 2,15 \\
\hline ALE-SP & 100,5 & 1,71 \\
\hline
\end{tabular}


Não há especificação de teor para cápsulas de alendronato de sódio. Com isso, foi levada em consideração a especificação adotada para comprimidos, a qual recomenda não menos que $90 \%$ e não mais que $110 \%$ de alendronato de sódio na formulação (24). Sendo assim, todos os lotes encontraram-se dentro do especificado e os valores de DPR obtidos entre as determinações foram relativamente baixos (menores que 3\%). Estes resultados sugerem que as matérias-primas utilizadas para o preparo das cápsulas foram pesadas de acordo com as quantidades indicadas na Tabela 1, não evidenciando erros no preparo das formulações.

\section{Determinação do peso médio das cápsulas}

Para cápsulas duras que apresentam peso médio de $300 \mathrm{mg}$ ou mais deve ser considerado o limite variação de $\pm 7,5 \%$, podendo ser aceitas até duas unidades aci- ma ou abaixo desta especificação. No entanto, nenhuma cápsula deve estar acima ou abaixo do dobro da porcentagem indicada, ou seja, $15 \%$ (23).

Os valores de peso médio encontrados para as formulações analisadas e os valores extremos obtidos, em comparação aos limites farmacopeicos, estão relacionados na Tabela 3.

Os resultados para os pesos individuais das cápsulas encontraram-se dentro das especificações, pois somente a formulação ALE-SP apresentou apenas uma unidade acima do limite superior de $7,5 \%$, sem, no entanto, superar o dobro deste porcentual (Tabela 3).

$\mathrm{O}$ desvio padrão relativo dos valores de peso encontrados para as formulações ALE-A, ALE-SP-T e ALE-SP também foi determinado, apresentando os seguintes valores, respectivamente: $2,4 \%, 2,9 \%$ e $3,8 \%$.

Tabela 3 - Valores de peso (em mg) obtidos para as cápsulas em cada método de homogeneização.

\begin{tabular}{|c|c|c|c|}
\hline Formulação & Peso médio \pm DP & Menor valor obtido (LI) & Maior valor obtido (LS) \\
\hline ALE-A & $324,0 \pm 7,7$ & $301,9(299,7)$ & $336,4(348,3)$ \\
\hline ALE-SP-T & $327,5 \pm 9,6$ & $305,8(302,9)$ & $344,7(352,0)$ \\
\hline ALE-SP & $325,1 \pm 12,2$ & $300,9(300,7)$ & $350,5^{*}(349,5)$ \\
\hline
\end{tabular}

Onde $n=40$ para cada formulação; DP = Desvio Padrão; LI e LS: Limite Inferior e Limite Superior, respectivamente, calculados com base no limite de variação de $\pm 7,5 \%$ em relação ao peso médio (Farmacopeia Brasileira $5^{\mathrm{a}}$ edição). *Único valor experimental acima do limite farmacopeico.

Considerando os valores de DPR obtidos e os gráficos da determinação de peso (Figura 1), todas as formulações analisadas apresentaram distribuição do pó razoavelmente uniforme entre todas as cápsulas, demonstrando um eficiente e homogêneo processo de enchimento. Porém, pode-se perceber uma variabilidade maior para os pesos individuais das cápsulas dos lotes ALE-SP, o que poderia ser atribuído a um fluxo prejudicado da mistura de pós, fator este que afeta diretamente o enchimento das cápsulas pela técnica de nivelamento de superfície (13). O escoamento deficiente da mistura pode ser explicado pela irregularidade no tamanho das partículas $(3,25)$, já que para a produção destes lotes não foi realizada tamisação prévia das matérias-primas.

Visando melhorar o escoamento dos pós durante a manipulação, todas as formulações foram adicionadas de dióxido de silício coloidal (Aerosil ${ }^{\mathbb{}}$ ), o qual é um promotor de fluxo tradicionalmente empregado na produção de formas sólidas (1-3). Entretanto, a baixa energia do processo de homogeneização em saco plástico pode ter prejudicado a adequada distribuição do dióxido de silício coloidal na superfície das partículas, contribuindo para o fluxo prejudicado da mistura.

\section{Determinação da uniformidade de conteúdo das cápsulas}

Segundo as farmacopéias Brasileira e Americana $(23,24,26)$, na avaliação da uniformidade de conteúdo, 10 cápsulas devem ser analisadas quanto ao teor de substância ativa, individualmente. Os resultados encontrados devem ser avaliados empregando-se o "Valor de Aceitação" (VA), o qual deve ser calculado através da fórmula geral:

$$
\mathrm{VA}=|\mathrm{M}-\mathrm{X}|+\mathrm{k} \cdot \mathrm{s}
$$

Onde:

$M=$ Valor de referência, o qual depende da média dos limites especificados na monografia individual e da média dos conteúdos individuais;

$\mathrm{X}=$ Média dos conteúdos individuais;

$\mathrm{k}$ = constante de aceitabilidade, a qual depende do número de unidades testadas;

$\mathrm{s}=$ desvio padrão da amostra. 
O limite máximo permitido para o valor de aceitação é de 15,0. Além disto, nenhuma unidade individual deve ultrapassar os limites estabelecidos pelas fórmulas:

$$
\begin{aligned}
& \text { Limite inferior }=(1-\mathrm{L} 2 \times 0,01) \times \mathrm{M} \\
& \text { Limite superior }=(1+\mathrm{L} 2 \times 0,01) \times \mathrm{M}
\end{aligned}
$$

Onde:

$\mathrm{L} 2=25,0$ (este valor pode mudar, caso seja especificado de forma diferente na monografia individual).

Como pode ser observado nas Tabelas 4 e 5 , todos os lotes apresentaram resultados de acordo com as especificações, já que os valores de aceitação estão abaixo de 15 e nenhuma unidade mostrou-se abaixo do limite inferior ou acima do limite superior estabelecido.

Figura 1: Distribuição de peso médio em cápsulas de alendronato de sódio em diferentes formas de homogeneização

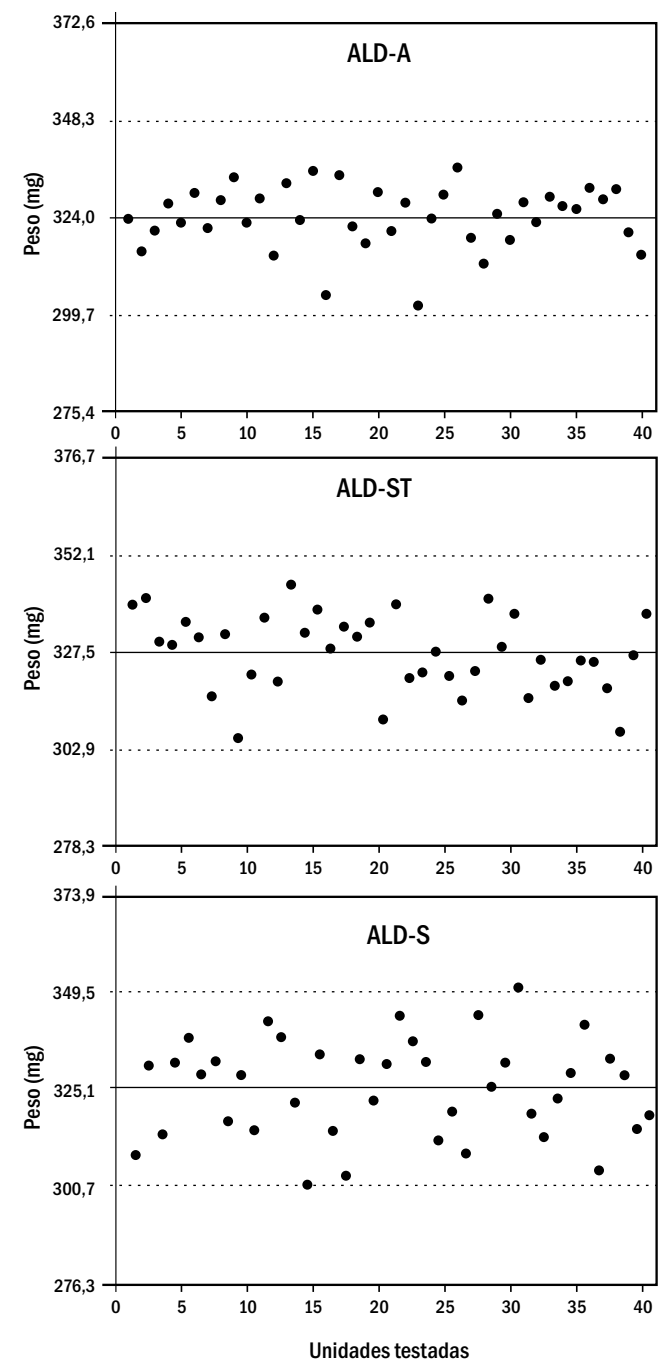

Tabela 4. Valores de aceitação encontrados para cápsulas de alendronato de sódio, em diferentes métodos de homogeneização.

\begin{tabular}{|c|c|c|c|c|c|c|}
\hline Formulação & Média & $\mathbf{n}$ & $\mathbf{K}$ & $\mathbf{s}$ & $\mathbf{M}$ & VA \\
\hline ALE-A & 102,9 & 48 & 2,00 & 2,7 & 101,5 & 6,8 \\
\hline ALE-SP-T & 100,4 & 60 & 2,00 & 3,4 & 100,4 & 6,7 \\
\hline ALE-SP & 100,4 & 53 & 2,00 & 5,0 & 100,4 & 9,9 \\
\hline
\end{tabular}

Tabela 5 - Valores de uniformidade de conteúdo (\%) encontrados para as cápsulas de alendronato de sódio, em diferentes métodos de homogeneização.

\begin{tabular}{|c|c|c|} 
Formulações & $\begin{array}{c}\text { Menor valor } \\
\text { obtido (LI) }\end{array}$ & $\begin{array}{c}\text { Maior valor } \\
\text { obtido (LS) }\end{array}$ \\
\hline ALE-A* & $97,0(76,1)$ & $109,6(126,9)$ \\
\hline ALE-SP-T** & $91,5(75,3)$ & $107,3(125,5)$ \\
\hline ALE-SP*** & $89,2(75,3)$ & $116,8(125,5)$ \\
\hline
\end{tabular}

Onde: $* \mathrm{n}=48 * * \mathrm{n}=60 * * * \mathrm{n}=53$; LI e LS: Limite Inferior e Limite Superior, respectivamente, calculados com base nas recomendações da Farmacopeia Brasileira $5^{\text {a edição }}$

Apesar de todas as formulações mostrarem resultados dentro das especificações da Farmacopeia Brasileira (23), a formulação ALE-SP apresentou maior valor de desvio padrão, indicando uma maior variabilidade nas doses das cápsulas obtidas através do método de homogeneização em saco plástico.

A Figura 2 mostra os gráficos de dispersão obtidos com os resultados da uniformidade de conteúdo das formulações analisadas. A formulação ALE-A apresentou menor dispersão dos resultados, mostrando uma melhor uniformidade de doses para as cápsulas produzidas através do método de homogeneização em almofariz. Por outro lado, com evidente maior variabilidade nos resultados de teor individual, a formulação ALE-SP apresentou menor uniformidade na distribuição das doses.

Após análise estatística dos dados (teste de Fisher para as variâncias), a formulação ALE-SP apresentou diferença significativa em relação aos outros lotes avaliados, como pode ser observado na Tabela 6. $\mathrm{O}$ valor $\mathrm{F}$ calculado foi menor que o valor $\mathrm{F}$ crítico apenas na comparação das variâncias das formulações ALE-A e ALE-SP-T, não demonstrando diferença estatisticamente significativa entre as variâncias obtidas para estes dois métodos de homogeneização.

Tais resultados sugerem que ocorre uma baixa eficiência de homogeneização quando as matérias-primas são diretamente adicionadas ao saco plástico e misturadas. Entretanto, a homogeneização em saco plástico utilizando matérias-primas previamente tamisadas parece proporcionar uma uniformidade semelhante à obtida em homogeneização com auxílio de gral e pistilo. 
O sucesso na produção de uma mistura homogênea depende de diversos fatores relacionados às características dos pós e também às forças atuantes durante a mistura. Para que o processo de homogeneização seja eficaz, é necessário que as forças de aceleração produzidas pelos movimentos do método de mistura superem as forças que tendem a manter as partículas adjacentes em uma posição fixa (25). Desta forma é possível provocar uma movimentação adequada dos componentes, o que pode resultar em diferentes mecanismos de mistura, como a convexão, quando grupos de partículas se deslocam de um local para outro do recipiente, a deslocação por deslizamento, quando há uma mudança na configuração dos componentes mediante o deslizamento da mistura, ou a difusão, quando o movimento desordenado das partículas provoca uma mudança de posição de cada partícula em relação à outra $(3,25)$. Geralmente, o mecanismo de difusão ocorre quando há forças de corte e impacto elevadas, levando à quebra de agregados de partículas, sendo assim mais eficaz em produzir misturas com menor grau de segregação (25). Possivelmente, os movimentos provocados pela mistura direta em saco plástico, na formulação ALE-SP, não forneceram energia suficiente para um adequado grau de homogeneização, enquanto os movimentos característicos da mistura em almofariz produziram maior força de corte e impacto, privilegiando a mistura por mecanismo de difusão e resultando em maior uniformidade para a formulação ALE-A.

Entre as características dos pós que podem afetar a qualidade da formulação com relação à segregação dos componentes destaca-se o tamanho de partícula (25).

Tabela 6 - Avaliação estatística dos resultados da uniformidade de conteúdo em cápsulas de alendronato de sódio preparadas por diferentes métodos de homogeneização

\begin{tabular}{|c|c|c|c|} 
Formulação & $\begin{array}{c}\mathbf{F} \\
\text { calculado }\end{array}$ & $\begin{array}{c}\mathbf{F} \\
\text { crítico }\end{array}$ & valor-P* \\
\hline ALE-A x ALE-SP-T & 1,54 & 1,59 & 0,1262 \\
\hline ALE-A x ALE-SP & 3,33 & 1,61 & $<0,0001$ \\
\hline ALE-SP-T x ALE-SP & 2,16 & 1,56 & 0,0044 \\
\hline
\end{tabular}

$* \alpha=0,05$.

Estudos mostraram que menores tamanhos de partícula para o fármaco tendem a resultar em misturas mais uniformes $(9,12)$. Uma correlação entre o grau de dispersão no tamanho das partículas e a uniformidade de conteúdo foi estabelecida por Yalkowsky e Bolton (1990), onde quanto maior o coeficiente de variação do diâmetro, menor o tamanho de partícula necessário para a obtenção de unidades farmacêuticas com $99 \%$ de probabilidade de passar no teste de uniformidade de conteú-
Figura 2: Uniformidade de conteúdo em cápsulas de alendronato de sódio em diferentes formas de homogeneização
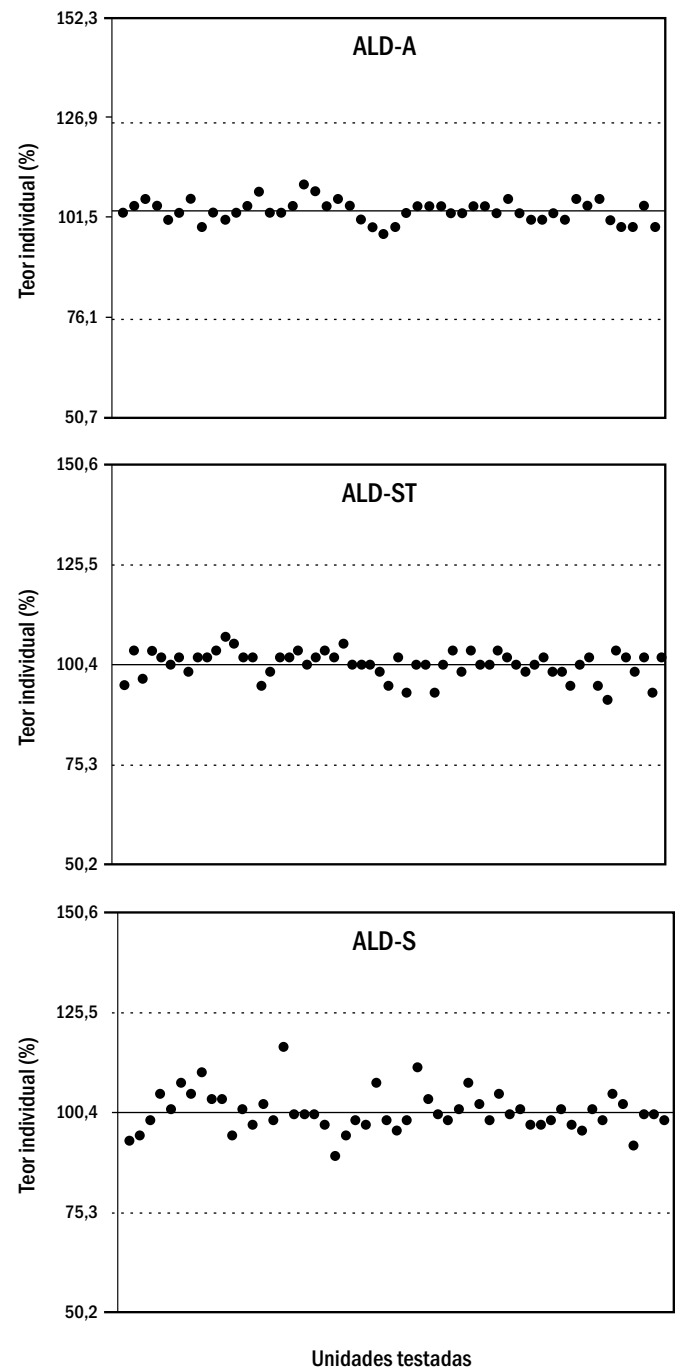

do, principalmente para doses menores do fármaco (12). Entretanto, embora menores diâmetros de partícula possam levar a misturas mais homogêneas, uma redução excessiva no tamanho pode levar à formação de agregados que dificultariam o processo de mistura (9). Na mistura em almofariz, os componentes são triturados ao mesmo tempo em que são homogeneizados, proporcionando uma redução e, também, uma regularização no tamanho das partículas. Este processo não se aplica à mistura em saco plástico, onde as partículas dos componentes permanecem com tamanho irregular e supõe-se que por esta razão possam segregar-se antes ou mesmo no momento do enchimento, prejudicando a uniformidade da mistura no lote ALE-SP. Por outro lado, a tamisação das matérias-primas previamente ao processo de mistura em saco plástico, que proporciona uma maior uniformização no tamanho das partículas dos componentes, evita a 
segregação dos pós na mistura, possibilitando uma homogeneização eficiente para o lote ALE-SP-T, mesmo quando se utiliza um método de baixa energia como o saco plástico.

Além disto, como foi observado por Pinheiro e colaboradores (2008), pode haver uma relação direta entre a variação de peso e a uniformidade de doses, principalmente para cápsulas com elevado DPR na determinação de peso (16). Uma mistura com partículas de tamanhos diferentes tende a apresentar um escoamento deficiente e prejudicar o enchimento das cápsulas, o que também pode ter contribuído para a baixa uniformidade de conteúdo da formulação ALE-SP.

Sendo assim, o emprego do método do saco plástico para o preparo de formulações sólidas deve ser considerado com cautela, principalmente quando não há padronização no tamanho das partículas dos componentes, já que pode levar à produção de misturas sem homogeneidade, prejudicando a uniformidade de doses e a qualidade do medicamento.

\section{REFERÊNCIAS}

1. Ferreira AO. Guia Prático da Farmácia Magistral. 3a. ed. São Paulo: Pharmabooks. 2008.

2. Ansel HC, Popovich NG, Ellen LV. Formas farmacêuticas e sistemas de liberação de fármacos. 8a. ed. Porto Alegre: Artmed. 2007.

3. Prista LN, Alves AC, Morgado R, Lobo JMS. Tecnologia farmacêutica. 6a. ed. Vol. 1 e Vol. 2. Lisboa: Fundação Calouste Gulbenkian. 2002

4. Ferreira, A. M. N.; Volpato, N. M.; Freitas, Z. M. F., Costa VA, Barros CM. Chiavegatto LFS, Quaresma CH. Validação do Processo de Mistura e Armazenamento de Pós para o Preparo de Cápsulas de Hidroxizine $25 \mathrm{mg}$. ANFARMAG - Revista do Setor Farmacêutico Magistral. 2003. 43:88-90.

5. Kornchankul W, Hamed E, Parikh NH, Sakr A. Effect of drug proportion and mixing time on the content uniformity of a low dose drug in a high shear mixer. Pharmazie. 2002. 57(1):49-53.

6. Swaminathan V, Kildsig DO. Effect of magnesium stearate on the content uniformity of active ingredient in pharmaceutical powder mixtures. AAPS Pharm. Sci. Tech. 2002. 3(3):27-31.

7. Venables HJ, Wells JI. Powder Mixing. Drug. Dev. Ind. Pharm. 2001. 27(7):599-612.

8. Prescott JK, Garcia TP. A solid dosage and blend content uniformity troubleshooting diagram. Pharmaceutical Technology. 2001. 25(3):68-88.

9. Zhang Y, Johnson KC. Effect of drug particle size on content uniformity of low-dose solid dosage forms. Int. J. Pharm. 1997. 154(2):179-183.

\section{CONCLUSÃO}

Para se garantir a qualidade das cápsulas produzidas em farmácias magistrais, o método escolhido para a manipulação da formulação é extremamente importante, sendo necessário adotar uma técnica de mistura padronizada e cuja eficiência possa ser comprovada.

Como esperado, a técnica de mistura em almofariz demonstrou ser o método mais eficaz para a homogeneização da mistura de pós, pois apresentou os melhores resultados para a uniformidade de conteúdo. Já o método utilizando saco plástico, apesar de prático, possui baixa energia, produzindo cápsulas com elevada variabilidade e baixa uniformidade das doses. A mistura em recipiente plástico pode ser considerada de forma segura, desde que se garanta previamente a regularização do tamanho das partículas dos componentes da formulação através, por exemplo, do procedimento de tamisação das matérias-primas

10. Carstensen JT, Dali MV. Blending Validation and Content Uniformity of Low-Content, Noncohesive Powder Blends. Drug. Dev. Ind. Pharm. 1996. 22(4):285-290.

11. Berman J, Planchard JA. Blend Uniformity and Unit Dose Sampling. Drug. Dev. Ind. Pharm. 1995. 21(11):1257-1283.

12. Yalkowsky SH, Bolton S. Particle Size and Content Uniformity. Pharm. Res. 1990. 7(9):962-966.

13. Petry RD, De Souza TP, Da Silva FA, Heberlé G, Da Silva WB, Fleck JD, Bassani VL, González-Ortega G, Petrovick PR, Guterres SS. Influência de adjuvantes e técnica de enchimento sobre as características farmacêuticas de cápsulas de gelatina dura contendo teofilina. Caderno de Farmácia. 1998. 14(1/2):13-19.

14. Staniforth JN, Rees JE. Effect of vibration time, frequency and acceleration on drug content uniformity. J. Pharm. Pharmacol. 1982. 34(11):700-706.

15. Brasil. Ministério da Saúde. Agência Nacional de Vigilância Sanitária. Resolução RDC n ${ }^{\circ} 67$, de 8 de Outubro de 2007. Dispõe sobre Boas Práticas de Manipulação de Preparações Magistrais e Oficinais para Uso Humano em farmácias. Diário Oficial da União, Brasília, DF, 09 out. 2007.

16. Pinheiro GM, Benavide VG, Volpato NM, Cabral LM, Santos EP Indicadores para controle de processo na manipulação de cápsulas em farmácia. Rev. Bras. Farm. 2008. 89(1):28-31.

17. Cordeiro MP, Reis FA, Nogueira GB, Giglio CA, Carvalho PTC, Belchior ACG. O uso do alendronato de sódio na osteosporose. ConScientiae Saúde. 2009. 8(2):309-315. 
18. Silva P. Farmacologia. 7a. ed. Rio de Janeiro: Guanabara Koogan. 2006.

19. Moreno AH, Pezza HR, Pezza L. Potenciometric determination of alendronate in pharmaceutical formulations. Chem. Anal. 2004. 49:351-357.

20. Bueno F, Bergold AM. Desenvolvimento de técnicas para controle de qualidade de fármacos em farmácias de manipulação: metodologias para análise de alendronato de sódio e sua determinação em formulações farmacêuticas. Acta Farm. Bonaerense. 2000. 19(2):129-132.

21. Ribeiro AF, Volpato NM. Alendronato de sódio: metodologias para análise quantitativa. Quim. nova. 2005. 28(5):852-858.
22. Ribeiro AF. Análise titulométrica e cromatográfica de alendronato de sódio - matéria-prima e produto acabado. [Dissertação]. Rio de Janeiro: Faculdade de Farmácia, Universidade Federal do Rio de Janeiro. 2005.

23. Brasil. Agência Nacional de Vigilância Sanitária. Farmacopeia Brasileira - volume 1. 5a. ed. Brasília (DF), 2010.

24. United States Pharmacopeia. General Chapters. 30. ed. Rockville: United States Pharmacopeial Convention, 2007.

25. Lachman L, Lieberman HA, Kanig JL. Teoria e Prática na Indústria Farmacêutica. 3a. ed. Vol. 1. Lisboa: Fundação Calouste Gulbenkian. 2001.

26. Roesch GC, Volpato NM. A harmonização da avaliação farmacopeica da uniformidade de doses unitárias de medicamentos. Infarma. 2010. 22(1/4):3-15. 